\title{
Risiko oder Gefahr? - Die Bedeutung der Wahrnehmung von Katastrophen für ihre wirtschaftliche und rechtliche Bewältigung
}

\section{Einleitung}

Der wirtschaftliche Gegenstand des Versicherungsgeschäfts sind Risiken wie die eventuelle Zerstörung eines Hauses durch Feuer, Körper- und Sachschäden bei Verkehrsunfällen oder der Untergang einer Schiffsfracht im Sturm. Im Recht existieren diese Risiken in Form von Beschreibungen in den Versicherungsverträgen. Dabei kommt es nicht nur auf möglichst präzise Definitionen der Schadensereignisse an, bei deren Eintritt der Versicherer zu einer Ersatzzahlung verpflichtet sein soll, sondern auch auf die Haftungsausschlüsse für bestimmte Fälle, in denen der Versicherer nicht haften will. Ein aktuelles Beispiel dafür sind die Freizeichnungsklauseln, die durch Terroranschläge verursachte Schäden an Gebäuden und Mobiliar, Leib und Leben sowie durch Unterbrechungen von Geschäftstätigkeit (dies war in New York der größte Posten) vom Schutz der Versicherungsverträge ausnehmen. Derartige Regelungen sind historisch nicht neu ${ }^{1}$, haben aber erst nach den Anschlägen in New York am 11. September 2001 weltweite Verbreitung gefunden. ${ }^{2}$ Damals reagierte die Versicherungswirtschaft auf ein bestimmtes Katastrophenereignis. Viele Unternehmen entschieden sich für Haftungsausschlüsse, weil sie annahmen, dass ein Schaden wie der erlebte erneut verursacht werden könnte und dass sie den daraus erwachsenden wirtschaftlichen Belastungen nicht standhalten würden. In ihren Reaktionen spiegelte sich ihre Wahrnehmung des Schadensereignisses und ihre Vorstellung von der bestehenden Gefährdungslage genau wider.

Ähnliches konnte man auch nach dem verheerenden Erdbeben beobachten, das im Jahr 1906 San Francisco getroffen hatte. Damals teilte sich die internationale Versicherungswirtschaft in zwei Lager. Vor allem amerikanische und britische Unterneh-

1 Beispielsweise gab es Anfang des 20. Jahrhunderts in den Lebensversicherungspolicen verschiedener europäischer Anbieter Ausschlussklauseln hinsichtlich der »Anarchistengefahr«.

2 Beispiele bei R. P. Hartwig, September 11, 2001: The First Year. One Hundred Minutes of Terror that Changed the Global Insurance Industry Forever, New York 2002, S. 9 ff. (pdf: www.iii.org). Die Terrorattacke verursachte Versicherungskosten in Höhe von über 30 Mrd. Dollar und gilt als das größte Schadensereignis der Versicherungsgeschichte. Versicherungen gegen Terrorattacken werden in den USA derzeit mit staatlicher Unterstützung angeboten, obgleich erhebliche Argumente gegen eine Kalkulierbarkeit derartiger Angriffe sprechen; siehe H. Kunreuther / E. Michel-Kerjan, Insurability of (Mega-) Terrorism Attacks, Report for the OECD Task Force on Terrorism Risk, Paris 2004, sowie R. P. Hartwig / G. Stewart / C. Wilkinson, Terrorism, Insurance and the United States Government, New York 2004 (pdf: www.iii.org). - Dagegen reagierte die Versicherungswirtschaft auf die Tsunami-Katastrophe vom 26. Dezember 2004 nicht mit einer verstärkten Anwendung von Ausschlussklauseln. Zum einen erwiesen sich die zu ersetzenden Schäden mit maximal 4 Mrd. Dollar als weniger umfangreich als zunächst erwartet. Zum anderen gelten TsunamiEreignisse als kalkulierbar. Insurance Information Institute (Hrsg.), Asian Earthquake and Tsunami. An Insurance Perspective, March 29, 2005 (pdf: www.iii.org); Swiss Re (Hrsg.): Tsunami in Südasien: Finanziellen Schutz aufbauen, Zürich 2005 (pdf: www.swissre.com). 
men bemühten sich darum, Erdbebenfolgen als zusätzliche Risiken in ihre Verträge zu integrieren. Viele kontinentaleuropäische Versicherer forderten demgegenüber, die Erdbebengefahr in juristisch einwandfreier Weise auszuschließen.

In der historischen Perspektive erweist sich der damalige Diskurs über den Umgang mit Erdbeben als Auslöser von Stadtkatastrophen als der erste bewusste Versuch, global einheitlich mit wirtschaftlichen und rechtlichen Mitteln auf eine veränderte Wahrnehmung einer bestimmten Bedrohungslage zu reagieren. ${ }^{3}$

\section{Die Erdbeben-Feuer-Katastrophe von San Francisco (1906)}

Am 18. April 1906 erschütterte ein Erdbeben der Stärke 8,3 auf der nach oben offenen Richterskala die kalifornische Metropole San Francisco. Sofort brachen einige Dutzend Brände aus. Hunderttausende Menschen flohen aus der Stadt. Die Feuerwehr war machtlos, da das Beben auch die Löschwasserleitungen zerstört hatte. Drei Tage lang wüteten die Feuerstürme. Der Augenzeuge Jack London fasste ihr Werk in einem einzigen Satz zusammen: »San Francisco is gone. «

Im Sommer und Herbst des Jahres 1906 musste die internationale Versicherungswirtschaft etwa zwei Drittel des Gesamtschadens ersetzen. ${ }^{5}$ Betroffen waren in erster Linie amerikanische, britische und mitteleuropäische Feuer- und Rückversicherungsgesellschaften. Fast alle Versuche, die Ansprüche auf Schadensersatz abzuwehren, scheiterten. In den meisten Verträgen fehlten juristisch einwandfreie Klauseln, die eine Haftung bei derartigen Katastrophen ausgeschlossen hätten. ${ }^{6}$ Vertreter der Versicherten von San Francisco klagten Millionensummen ein - nicht nur in den USA, sondern auch vor deutschen, österreichischen und schweizerischen Gerichten.

3 Der vorliegende Aufsatz vertieft einen Aspekt aus der Dissertation des Verfassers, die voraussichtlich Ende 2005 unter dem Titel »Rechtsbildung im wirtschaftlichen »Weltverkehr«. Die internationale Standardisierung von Vertragsbedingungen (1871-1914)« erscheinen wird.

4 J. London: The Story of an Eyewitness, in: Collier's Weekly vom 5. Mai 1906.

5 Nach heutigem Kenntnisstand betrug der materielle Gesamtschaden mehr als 400 Millionen Dollar. Siehe z.B. J. R. Freeman, Earthquake Damage \& Earthquake Insurance (1932), S. 663.

6 Typische, in Kalifornien verbreitete Formulierungen des Gefahrenausschlusses lauteten:

1. Fallen building clause (Einsturzklausel): »If a building or any part thereof fall, except as the result of fire, all insurance on such building or its contents shall immediatly cease." Diese Klausel umfasste nach früherer Gerichtspraxis nur Schadensfälle, in denen ein integraler oder umfänglicher Teil des Gebäudes zusammengebrochen war. Dies konnten die Versicherer vor Ort fast nie belegen. Einsturzklausel zit. nach A. W. Whitney: Report of the special committee of the Board of trustees of the Chamber of Commerce of San Francisco on the insurance settlements incident to the 1906 San Francisco fire, S.F., Cal. 1906, S. 39.

2. Earthquake clause (Erdbebenklausel, Beispiel): »This company shall not be liable for loss caused directly or indirectly by invasion, insurrection [...]; or for loss or damage occasioned by or through any volcano, earthquake, $[\ldots] . \ll$ Hier ist der Ausschluss der Haftung des Versicherers für erdbebenverursachte Brandschäden deutlicher formuliert. Im Einzelfall stellte sich jedoch oft die Frage, ob sie für Schäden galt, die erst einen oder zwei Tage nach dem Erdbeben - etwa durch Überspringen des Feuers - entstanden waren. Außerdem gelang es nur wenigen Versicherern, den schwierigen Beweis der Kausalität zwischen Erdbeben und konkretem Schaden zu erbringen. Erdbebenklausel gekürzt zit. nach: A. W. Whitney a.a.O., S. 37 f. 


\section{Die Wahrnehmung der Versicherungswirtschaft}

Aufgrund dieser Erfahrungen versuchten viele der direkt oder indirekt betroffenen Versicherungsgesellschaften, sich für die Zukunft gegen die wirtschaftlichen Folgen derartiger Katastrophen abzusichern. Ihre Reaktionen fielen sehr unterschiedlich aus. Dies lag nur teilweise daran, dass sich die Situation der Versicherungsunternehmen nach dem Erdbeben sehr uneinheitlich darstellte. Die Verschiedenheit der Reaktionen beruhte auch auf Unterschieden in der Wahrnehmung der katastrophalen Ereignisse ${ }^{7}$ und in der Vorstellung von Seismizität. Sie führte die private Versicherungswirtschaft in eine Spaltung, die sich bereits im Jahr 1906 abzeichnete: Ein Teil der Versicherer hielt erdbebenverursachte Feuerschäden für versicherbar; der andere Teil nicht.

\section{San-Francisco-Katastrophen. Unterschiede in der Wahrnehmung des Geschehenen}

Worin bestand die Katastrophe von San Francisco? Für die Bewohner der Stadt, für ihre Wirtschaft und ihre Verwaltung waren es der Tod tausender Menschen und die Vernichtung fast aller materiellen Werte durch den Feuersturm. In der Wahrnehmung der Versicherer hingegen wurde »San Francisco « zum Synonym für eine wirtschaftliche Katastrophe. Die zeitgenössischen Äußerungen aus Versicherungskreisen zeigen, dass viele die gesamte politische Situation in Kalifornien einschließlich des Verhaltens der Geschädigten, der Behörden und der Gerichte als Teil der Katastrophe empfanden.

Dementsprechend divergierten die Auffassungen über die Ursache der Ereignisse. Die Bewohner von San Francisco folgten bereitwillig einer Umdeutung, bei der das Erdbeben als zentraler Faktor verschwand und durch den Massenbrand ersetzt wurde. Diese Interpretation hatten lokale Wirtschaftskreise und Politiker gezielt gestreut, um die Öffentlichkeit davon zu überzeugen, dass die Erdbebengefahr an der Pazifikküste gering sei. Auf diese Weise versuchten sie, das günstige Geschäftsklima an der Westküste zu erhalten. ${ }^{8}$

In den Diskursen der Versicherungswirtschaft nahm das Erdbeben aus zwei Gründen sehr viel mehr Raum ein. Zum einen waren die meisten Unternehmen daran interessiert, den Ersatzansprüchen ihrer Versicherten substantiierte Einwendungen entgegensetzen zu können. Diese ließen sich am ehesten mit den Wirkungen des Erdbebens begründen. Zum anderen gehörte die Untersuchung von Schadensursachen zu den essentiellen Grundlagen des Versicherungswesens. Nach der Katastrophe von San Fran-

7 Zur Wahrnehmung von Naturkatastrophen siehe D. Groh / M. Kempe / F. Mauelshagen, Naturkatastrophen - wahrgenommen, gedeutet, dargestellt, in: dies., Naturkatastrophen. Beiträge zu ihrer Deutung, Wahrnehmung und Darstellung in Text und Bild von der Antike bis ins 20. Jahrhundert (2003), S. 11 ff. sowie G. Massard-Guilbaud, Introduction - The Urban Catastrophe: Challenge to the social, economic, and cultural order of the city, in: dies. / H. L. Platt / D. Schott, Cities and Katastrophes (2001), S. 9 ff.

8 G. Hansen / E. Condon, Denial of Disaster, S. 108 ff., 112, 120; C. Strupp, »Nothing destroyed that cannot speedily be rebuilt", in: A. Ranft / S. Selzer, Städte aus Trümmern (2004), S. 165 ff.; T. Steinberg, Acts of God, S. 27 ff.; C.-H. Geschwind, California Earthquakes, S. $23 \mathrm{ff}$. 
cisco herrschte in der internationalen Versicherungswirtschaft das Bewusstsein, dass jedes Unternehmen Entscheidungen über seine künftige Geschäftspolitik treffen musste. Deshalb setzten sich die Managements und Direktionen intensiv mit den Gefahren oder Risiken auseinander, die sich in San Francisco verwirklicht hatten.

Bei der Analyse setzten sie allerdings unterschiedliche Schwerpunkte. In den amerikanischen Beiträgen erschien die San-Francisco-Katastrophe tendenziell als anthropogenes Ereignis. Das Journal of Commerce veröffentlichte beispielsweise eine Serie von Stellungnahmen, in denen die Frage der Brandsicherheit amerikanischer Großstädte im Vordergrund stand. Die Autoren - Manager großer Versicherungsunternehmen - forderten Verbesserungen der urbanen Bauweise und Infrastruktur. ${ }^{9}$ Die europäischen Versicherungskreise schenkten diesem Aspekt weniger Aufmerksamkeit. Im Mittelpunkt ihrer Überlegungen stand und blieb das Erdbeben. Die Europäer nahmen das Ereignis vor allem als Naturkatastrophe wahr.

\section{Gefahr oder Risiko? Unterschiede in der Vorstellung vom Möglichen}

Die Unterschiede zwischen der »amerikanischen« und der »europäischen« Sicht auf das Geschehen in San Francisco fanden eine Fortsetzung in der Frage, wie Erdbeben als Auslöser von Stadtbränden einzuschätzen seien. Konnten Erdbeben in das Feuerversicherungsgeschäft integriert werden? Handelte es sich also um Risiken, die wissenschaftlich erfasst, mathematisch kalkuliert und wirtschaftlich bewältigt werden konnten? Oder stellten Erdbeben unberechenbare Gefahrenquellen dar, von denen sich die Feuerversicherer fernhalten sollten? Die Beantwortung dieser Fragen war für Entscheidungen über die künftige Geschäftspolitik von zentraler Bedeutung.

Eine strenge begriffliche Unterscheidung zwischen »Gefahr« und »Risiko« entspricht nicht dem Sprachgebrauch der damaligen Zeit. ${ }^{10}$ Sie ist aber bei der Untersuchung der unterschiedlichen Vorstellungen von Erdbeben hilfreich, weil sie eine verborgene Trennlinie zwischen den späteren Befürwortern und Gegnern einer Ausschlussklausel offenlegt.

Als Gefahren werden Zustände wahrgenommen, in denen schädigende Ereignisse drohen. Bis in die Frühe Neuzeit hatte die Einschätzung von Gefahren auf zufällig gewonnenen Erfahrungen beruht. Systematische Untersuchungen waren selten erfolgt. Dementsprechend gering waren die Möglichkeiten gewesen, die Wahrscheinlichkeit des Eintritts bestimmter Ereignisse zu erfahren. ${ }^{11}$

Dies änderte sich in der Frühen Neuzeit. Mathematiker, Naturwissenschaftler und Ökonomen begannen, die Ursachen und Verläufe bestimmter Ereignisse und die Häufigkeit ihres Eintritts zu erforschen. Ihr Interesse galt Naturereignissen, der Sterblich-

9 Den Anstoß gab der Beitrag des Präsidenten der New Yorker Gesellschaft Continental, Henry Evans, The Earthquake Clause, in: The Journal of Commerce vom 25.1.1907, S. 13.

10 Der Begriff Risiko bzw. risk, risque, rischio, riesgo etc. wurde und wird häufig syonym für »Gefahr« und »Wagnis« verwendet. Eine interdisziplinäre Klärung ist bis heute nicht gelungen. Allein elf verschiedene Risikobegriffe unterscheiden G. Banse / G. Bechmann, Interdisziplinäre Risikoforschung. Eine Bibliographie (1998), S. 29 ff.

11 Die Idee der Wahrscheinlichkeitstheorie entstand in der Renaissance. Ihre Grundlagen wurden zwischen 1700 und 1760 entwickelt. P. L. Bernstein, Against the Gods (1996), S. 3 ff. 
keit bestimmter Bevölkerungsgruppen und ähnlichen Gegenständen. ${ }^{12}$ Mit Hilfe der Wahrscheinlichkeitsrechnung gelang es ihnen, die Unsicherheit über den künftigen Eintritt derartiger Ereignisse zu verringern. Praktische Anwendung fand diese Technik in staatlichen Verwaltungen und im Versicherungswesen, das sich zur gleichen Zeit über weite Lebensbereiche ausbreitete. ${ }^{13}$

Durch diese systematischen Untersuchungen von Schadensereignissen wurden »Gefahren « $\mathrm{zu} »$ Risiken « transformiert. Risiken unterscheiden sich von Gefahren dadurch, dass die Unsicherheit über den Eintritt der drohenden Ereignisse durch die Gewissheit einer bestimmten Wahrscheinlichkeit ersetzt wird. Risiken sind - im Gegensatz zu Gefahren - der Kalkulation zugänglich. Gerade dadurch konnten sie im Versicherungswesen zu Vertragsgegenständen werden. ${ }^{14}$

Das Risiko-Paradigma verbreitete sich in allen Gesellschaften, die sich im Industrialisierungsprozess befanden. ${ }^{15} \mathrm{Zu}$ Beginn des 20. Jahrhunderts bildete es ein unverzichtbares Fundament für die privaten und staatlichen Versicherungssysteme, die Finanzwirtschaft, die betriebswirtschaftliche Kalkulation und andere Bereiche.

Die Diskussionen der Versicherer über die Gefahr oder das Risiko von erdbebenverursachten Großbränden verliefen parallel zu ihren Diskussionen über die Ursachen der San-Francisco-Katastrophe. Sie bewegten sich auf zwei Ebenen: derjenigen der Technik und derjenigen der Seismologie.

Nach technischen Lösungen suchten vor allem die amerikanischen Versicherer. In zahlreichen Fachzeitschriften erschienen aktuelle Berichte, die sich mit der Bauweise, Architektur und Infrastruktur der Großstädte auseinandersetzten. ${ }^{16}$ Mehrere Kommissionen wurden mit der Untersuchung dieser Fragen beauftragt. ${ }^{17}$ Doch nachhaltige

12 Eine Voraussetzung dieser Forschungen war die Abkehr von methaphysischen Deutungen von Naturereignissen, die ab dem 17. Jahrhundert erkennbar ist. G. Massard-Guilbaud (wie Fn. 7), S. 21.

13 P. L. Bernstein, Against the Gods (1996), S. 86 ff.; B. Supple, The Royal Exchange Assurance (1970), S. $3 \mathrm{ff}$.

14 Der hier verwendete Begriff von Risiko, der dieses Konzept in den historischen Zusammenhang der Entstehung der Moderne stellt, entspricht demjenigen von P. L. Bernstein, Against the Gods (1996), S. 1 ff. Er unterscheidet sich von dem entscheidungstheoretisch geprägten Konzept der neueren Soziologie, das die Anthropogenität von Risiken betont. Für die historische Forschung erscheint diese Begrenzung nicht hilfreich.

15 Ein deutliches Indiz hierfür ist die starke und in allen Bevölkerungsklassen zu beobachtende Ausbreitung der Lebensversicherung in Europa und Nordamerika, die auch in anderen Weltregionen zumindest die an der europäischen Lebensweise orientierten Bevölkerungsteile erfasste.

16 Der Versicherungswissenschaftler A. W. Whitney kritisierte, die schlechte Bauweise sei kaum zu bekämpfen (»indifference of the American people to the enormous annual fire-loss «) und fordert anlässlich der Katastrophe von San Francisco, umzudenken. A. W. Whitney (wie Fn. 6), S. 53 f.

17 Die wichtigsten waren: Report of the Sub-Committee on Statistics to the Chairman and Committee on Reconstruction (San Francisco 1907, Archivalien der Bancroft Library, Berkeley); Municipal Report on the San Francisco Earthquake and Fire of April 1906 (1907); J. S. Sewell / F. Soulé and R. L. Humphrey, The San Francisco Earthquake and Fire of April 18, 1906 and their effects on structures and Structural Materials, U.S. Geological Survey Bulletin No. 324 (1907); S. A. Reed, The San Francisco conflagration of April, 1906: Special Report to the National Board of Fire Underwriters' Committee of the Twenty (1906, Bancroft Library, Berkeley); Notes on the Californian Earthquake. Reports by the State Board of Architects, and by the Home Fire Insurance Co. Ills., Eng. Rec. May 19, 1906. 
Verbesserungen blieben aus. ${ }^{18}$ So weit hatte allerdings auch nicht das Vertrauen der Versicherer gereicht. Weder Europäer noch Amerikaner gaben sich allein mit technischen Lösungen zufrieden. Um zu erfahren, ob Erdbebenereignisse vorhersehbar oder zumindest der Wahrscheinlichkeit nach einschätzbar seien, suchten sie nach wissenschaftlich gesicherten Erkenntnissen über die Seismizität. Konnte das Katastrophenrisiko für die Städte an der amerikanischen Westküste und in anderen Regionen kalkuliert werden?

Die Wahrscheinlichkeit des Eintritts seismischer Ereignisse wurde um die Wende zum 20. Jahrhundert vor allem anhand von historischen Statistiken eingeschätzt. ${ }^{19}$ Über die Ursachen von Erdbeben hingegen herrschte Streit. Seit Jahrzehnten untersuchten Wissenschaftler so unterschiedliche Faktoren wie Luftdruck und Temperatur, Nordlichter, Feuerkugeln und Sonnenflecken, Magnetismus und Elektrizität bzw. Galvanismus. ${ }^{20}$ Gerade nach der San-Francisco-Katastrophe hatten viele abenteuerliche Theorien und Spekulationen Konjunktur ${ }^{21}$ und verbreiten sich unter den Versicherern auf dem europäischen Kontinent. So ließ die französische Fachzeitschrift L'Argus die alte These von einem Zusammenhang zwischen seismischen und meteorologischen Phänomenen wieder aufleben. ${ }^{22}$ Das deutsche Wirtschaftsblatt Handel und Industrie überlegte, ob die Vulkane »Sicherheitsventile« der Erde bildeten - die nur momentan nicht vor Erdbeben schützten. ${ }^{23}$ Angesichts dieser Unsicherheiten verfestigte sich in den kontinentaleuropäischen Versicherungskreisen der Eindruck, dass Aussagen über künftige Erdbeben unmöglich seien. Nach ihrer Vorstellung waren Erdbeben keine kalkulierbaren Risiken. ${ }^{24}$ Vielmehr schien man »augenblicklich [...] in einer Periode seismischer und vulkanischer Veränderungen der Erdoberfläche zu leben, die alle Voraussagen und Vorausberechnungen einfach unmöglich machen. ${ }^{25}$

18 Von den 20.000 neuen Gebäuden, die in den nächsten drei Jahren entstanden, erfüllten nur 1.700 die neuen Sicherheitsvorschriften der Behörden. Diese wurden wenig später wieder gelockert. C. Strupp (wie Fn.8), S. 159.

19 Die damals aktuellste Aufstellung stammte von dem französischen Seismologen Fernand de Montessus de Ballore und wurde in der Versicherungszeitschrift La Semaine vom 23.12.1906, S. 8, veröffentlicht.

20 J. Roth, Über die Erdbeben (1882), S. 5; 28 ff. (ablehnend gegenüber diesen Theorien); R. Brauns, Vulkane und Erdbeben (1913), S. 156 ff. (ohne eigene Festlegung). Unter Galvanismus wurden elektromagnetische Spannungen verstanden.

21 Siehe beispielsweise die Studie des Geheimen Bergrats Prof. R. Brauns, Vulkane und Erdbeben (1913), S. $156 \mathrm{ff}$.

22 L'Argus, zitiert nach: Der Versicherungsfreund vom 1.10.1906, S. 1. Vgl. R. Röttger, Erdbeben (1889), S. $32 \mathrm{ff}$.

23 Noch immer Erdbebengefahr, in: Handel und Industrie, zitiert nach: Der Versicherungsfreund vom 20.2.1907, S. 4.

24 Vgl. die Stellungnahme der Rückversicherer in: Zur Katastrophe in San Francisco, in: Masius' Rundschau 1906, S. 224 ff.; Ruttke, Die Versicherung gegen Erdbeben und andere unmeßbare Gefahren, in: Mitteilungen für die öffentliche Feuerversicherungs-Anstalten 1909, S. 237 ff.; E. v. Liebig, Das deutsche Feuerversicherungswesen (1911), S. 87.

25 Noch immer Erdbebengefahr, in: Handel und Industrie, zitiert nach: Der Versicherungsfreund vom 20.2.1907, S. 4. Ähnlich Der Grenzbote vom 21.6.1906 und Zur Frage der Deckung von Erdbebenschäden, in: Berliner Börsen-Courier vom 24.2.1915, Beilage S. III. 
Ganz anders klangen die Ausführungen des amerikanischen Schadensliquidators Robert K. Mackenzie ${ }^{26}$, der Anfang 1907 in einem Vortrag vor britischen Versicherern auf die Ursachen des Erdbebens von San Francisco einging. Kenntnisreich legte er dar, wie der Erdstoß vom 18. April 1906 entlang des San-Bruno-Grabens ${ }^{27}$, der bis tief unter die Erdoberfläche reiche und von Norden nach Süden entlang der kontinentalen Küste führe, geotektonische Hemmnisse beseitigt habe. ${ }^{28}$ Mit diesen Ausführungen brachte Mackenzie seine Zuhörer auf den neuesten Stand der seismologischen Forschung in den Vereinigten Staaten. Bereits wenige Wochen nach dem Erdbeben hatten amerikanische Zeitschriften wie National Geographic, Scientific American, Popular Science Monthly und das Journal of Geology fundierte Analysen der Ereignisses veröffentlicht. ${ }^{29}$ Die amerikanischen Wissenschaftler gingen davon aus, dass Erdbeben wie jenes von San Francisco mit der Gebirgsbildung in Zusammenhang standen. ${ }^{30}$ Einige Forscher hatten sich sogar zu der zukünftigen Gefährdung Kaliforniens geäußert. Aufmerksam nahm die angloamerikanische Versicherungswelt zur Kenntnis, dass in Kalifornien nach der Beseitigung der geotektonischen Spannungen bis zum nächsten Erdbeben viele Jahrzehnte, vielleicht ein Jahrhundert, vergehen würden. ${ }^{31}$

Der Gegensatz zwischen diesen kohärenten Vorstellungen amerikanischer Fachleute $^{32}$ über die Ursachen von Erdbeben und den oben geschilderten Unsicherheiten kontinentaleuropäischer Autoren ist frappierend. Amerikaner und Briten konnten die Möglichkeit von Erdbeben durchaus als kalkulierbares Risiko wahrnehmen. ${ }^{33}$ Der Informationsstand auf dem europäischen Kontinent hingegen reichte dafür nicht aus. ${ }^{34}$

26 R. K. Mackenzie arbeitete zu dieser Zeit in San Francisco an der Untersuchung und Taxierung von Schäden, die Versicherte der State Fire Insurance Co. (Liverpool) erlitten hatten.

27 Die heutige Bezeichnung ist San Andreas Fault. Neuere Kenntnisse über das Beben von 1906 faßt W. L. Ellsworth zusammen, Earthquake History, 1769-1989, in: R. E. Wallace (Ed.), The San Andreas Fault System (1990), S. 1159 f.

28 R. K. Mackenzie, The San Francisco earthquake \& conflagration (1907).

29 Vgl. F. L. Ransome (U. S. Geological Survey), The Probable Cause of the San Francisco Earthquake, in: The National Geographic Magazine of May, 1906, vol. XVII, No. 5; The San Francisco Earthquake, in: Scientific American vom 19.5.1906, S. 419 f.; und D. S. Jordans bedeutende Aufsatzsammlung The California Earthquake of 1906 (1907).

30 F. L. Ransome, The Probable Cause of the San Francisco Earthquake, in: The National Geographic Magazine, vol. XVII/No. 5, May 1906; ähnlich G. K. Gilbert, The Investigation of the California Earthquake, in: D. S. Jordan, The California Earthquake of 1906 (1907).

31 R. K. Mackenzie, The San Francisco earthquake \& conflagration (1907); C. Derleth, The Destructive Extent of the California Earthquake, in: D. S. Jordan, The California Earthquake, S. 100 f.; D. S. Jordan, The Earthquake Rift of April, 1906, in: ders., The California Earthquake, S. 55.

32 Allerdings wurden auch in den USA noch einige bereits aus damaliger wissenschaftlicher Perspektive veraltete Erklärungen verbreitet. D. R. Dean, The San Francisco Earthquake of 1906, in: Annals of Science 50 (1993), S. 511 ff.; G. Thomas/ M. Witts, The San Francisco Earthquake, 167f., 219.

33 Gleichwohl gab es Gegenstimmen und Meinungsverschiedenheiten, mitunter sogar innerhalb einzelner Versicherungsgesellschaften; dies schildert z.B. C. Trebilcock, Phoenix Assurance and the Development of British Assurance II (1998), S. $277 \mathrm{ff}$.

34 Mackenzies Äußerungen wurden 1907 auch in deutscher Sprache publiziert, blieben dort aber eine unter zahlreichen, unterschiedlichen Stimmen. 


\section{San Francisco, Valparaiso, Kingston: Die globale Dimension}

Noch während die Versicherer sich intensiv mit der Situation in Kalifornien auseinandersetzten, brachten ihnen weitere Erdbeben die globalen Ausmaße der seismischen Gefährdung vor Augen.

Am 16. August 1906 traf ein Erdbeben die chilenischen Provinzen Valparaiso und Talca. ${ }^{35}$ Bereits am selben Tag brannten mehrere Häuserblocks nieder. Weitere Brände, auch in anderen Orten der Region, folgten am 17. sowie am 20. und 21. August. Valparaiso, mit 125.000 Einwohnern eine der größten Städte der chilenischen Republik, wurde fast vollständig vernichtet.

Ein weiteres Erdbeben verwüstete am 19. Januar 1907 Kingston auf Jamaica. Zu dieser Zeit lebten etwa 46.000 Menschen in der britischen Kolonialhafenstadt. Auch hier folgten dem Beben tagelange Brände, die zahlreiche versicherte Gebäude zerstörten. ${ }^{36}$

Die Versicherer erlebten ihr zweites und ihr drittes »San Francisco«. Immer wieder stürzten die Aktienkurse der in Übersee tätigen Versicherungsgesellschaften an den internationalen Börsen ab. ${ }^{37}$ Immer wieder mussten sie erhebliche Summen an Schadensersatz leisten. In allen Ländern kam es zu juristischen Auseinandersetzungen um die Frage der Haftung der Versicherer. Zwar reichten die Ausmaße der späteren Katastrophen nicht an jene von San Francisco heran. ${ }^{38}$ Doch der Schock saß tief. Ein Zitat aus der Zeitschrift für die gesamte Versicherungswissenschaft brachte die Stimmung der Beteiligten auf den Punkt:

»Das Feuerversicherungswesen aller Kulturländer ist durch die wiederholten Erdbebenkatastrophen des letzten Jahres in seinen Grundfesten erschüttert worden und zwar weniger direkt durch die großen Verluste, die es durch Aufbringung der Schadenssummen erlitten hat, als indirekt durch die unheimliche Wahrnehmung, dass die Klauseln, mit deren Hilfe der Versicherer das Erdbebenrisiko von der Feuerversicherung ausschließen wollte, in der Praxis sich als unzulänglich erwiesen haben. ${ }^{39}$

35 In der Östereichische[n] Revue 1906, S. 213, erscheint Valparaiso als »die reiche, blühende, paradiesisch gelegene Perle Chiles «.

36 Zeitgenössische Berichte lieferten M. Hall, Earthquakes in Jamaica (1907); F. Treves, The Cradle of the Deep (1908); W. R. Hall Caine, The Cruise of the Port Kingston (1908); The Jamaica Gleaner, Geography and History of Jamaica ${ }^{24}$ (1995).

37 Betroffen waren neben chilenischen v.a. englische und in geringerem Ausmaß deutsche Gesellschaften. Österreichische Revue 1906, S. 213; Die Erdbebenkatastrophe von Valparaiso, in: Östereichische Versicherungs-Zeitung 1906, S. $252 \mathrm{f}$.

38 Berichte aus Valparaiso: Désastre du Chili, in: La Semaine vom 9.9.1906, S. 6; Die Erdbebenkatastrophe von Valparaiso, in: Östereichische Versicherungs-Zeitung 1906, S. 259; Feuerversicherung und Feuerschutz 1906, S. 227. Berichte aus Kingston: Feuerschaden durch Erdbeben, in: Der Versicherungsfreund vom 10.9.1907, S. 8; M. Herzog, Was Dokumente erzählen können, Bd. II, S. 270; R. A. Lobdell, The Jamaica Earthquake of 1907 (1993), S. $15 \mathrm{ff}$.

39 J.-D. v. Witzleben, Die Erdbebenklausel in den Feuerversicherungsverträgen, in: ZVersWiss 7 (1907), S. 378. 


\section{Wirtschaftliche und rechtliche Reaktionen}

\section{Zusatzprämie oder Erdbebenklausel?}

Die entscheidenden Weichen für den zukünftigen Umgang der Feuer- und Rückversicherer mit Erdbeben-Feuerschäden wurden noch im Lauf den Jahres 1906 gestellt. Die Unternehmensführungen mussten sich entscheiden, ob sie künftig für diese Art von Schäden aufkommen wollten oder nicht. In diese Entscheidung spielten viele Faktoren hinein. Von großer Bedeutung war die jeweilige Wahrnehmung der Seismizität als unberechenbare Gefahr oder als kalkulierbares Risiko. Außerdem beeinflussten die geschäftlichen Perspektiven in den Krisenregionen die Entscheidungen.

An den Kontroversen über den künftigen Umgang mit dem Erdbebenrisiko beteiligten sich europäische und nordamerikanische Versicherer sowie interessierte Kreise aus Wirtschaft, Politik und Administrationen. In Deutschland und in England, deren Versicherungswirtschaft in besonderem Maße an dem Ersatz der Schäden in Kalifornien beteiligt war, erreichten die Diskussionen große Intensität. Auch in den Niederlanden war ein starkes Interesse zu verzeichnen, das auf dem erheblichen Engagement der holländischen Gesellschaften in Übersee beruhte. In anderen, notorisch erdbebengefährdeten Ländern wie Italien wurde das Thema erstaunlicherweise kaum erörtert.

Die nationalen Diskussionen verliefen nicht scharf voneinander getrennt. Die Feuerund Rückversicherer informierten sich und einander aus der internationalen Tagesund Fachpresse. ${ }^{40}$ Es wurde dermaßen grenz- und staatenübergreifend argumentiert und reagiert, dass von einem internationalen Diskurs gesprochen werden kann. Gleichwohl verblieb der Meinungsaustausch zu dezentral, als dass sich gemeinsame Linien hätten herauskristallisieren können.

Dass eine neue Orientierung über den Umgang mit erdbebenverursachten Feuerschäden anstand, erkannten viele. Die Alternativen zu einem Rückzug der Gesellschaften aus allen gefährdeten Zonen lauteten: Übernahme des Erdbebenrisikos gegen hohe Zusatzprämien und unter bestimmten Restriktionen oder Einführung einer strengen Ausschlussklausel. ${ }^{41}$

Die Vorstellung von einer Übernahme des Erdbebenrisikos stieß vor allem unter den Rückversicherern auf heftige Ablehnung:

»Erdbeben ebenso wie vulkanische Eruptionen sind Naturereignisse, gegen welche vernünftigerweise eine Versicherung nicht gewährt werden kann, da das hierin liegende Risiko ein grenzenloses ist, grenzenlose Verpflichtungen aber Versicherungsgesellschaften selbst für einzelne Gebiete nicht übernehmen dürfen, weil sie sich hier-

40 Dies geht aus den Korrespondenzen beteiligter Direktoren hervor, z.B. aus dem Schreiben von H. Grünwald (Kölnische Rückv.-G.) an C. Simon (Schweizerische Rückv.-G.) vom 14.9.1906, Archiv der Swiss Re (Zürich) Nr. 9.0-29/ Mappe III/ S.F. 18.4.1906/ Diverse Corresp. (v.a. Rückv. 6/1906-6/1907), Bl. 183 f.; sowie aus der Antwort Simons an Grünwald vom 15.9.1906, Archiv der Swiss Re a.a.O., Bl. 185 f.

41 F. Lock, Amerikanische Feuerversicherungsmethoden und Bedingungen, in: Insurance World von Oktober 1906, übersetzt in: Der Versicherungsfreund vom 1.11.1906, S. 2.; The Earthquake Clause, in: The Adjuster 33, Oktober 1906, S. 189 f. 
durch der Gefahr aussetzen würden, ihren Pflichten den Versicherten gegenüber nicht oder nur teilweise nachkommen zu können. «2

Mit diesen Worten begründete ein Vertreter der Münchener Rückversicherungs-Gesellschaft gegenüber der Frankfurter Zeitung seine Auffassung, die Übernahme von Erdbebenrisiken sei »Wagemut«. Nach seinen Angaben entsprach der in San Francisco geleistete Schadensersatz einem Betriebszeitraum von 900 Jahren; dem Erdbeben-Feuerrisiko entsprechende Prämien müssten also »exorbitant hoch « veranschlagt werden. ${ }^{43}$

Gesucht wurde statt dessen nichts weniger als die perfekte »Erdbebenklausel«. Dieses strategische Ziel hatte sich bereits auf der internationalen Rückversicherungskonferenz am 30. April 1906 in Frankfurt am Main durchgesetzt. In ihrer Resolution bezeichneten die Rückversicherer es als »wünschenswert, um auch den Versicherten die klare Kenntnis der Situation zu vermitteln, dass künftighin in sämtlichen Policen in irgend einem Gebiet, wo solches noch fehlt, eine Klausel alsbald zur Aufnahme gebracht wird, welche die Ersatzpflicht für allen und jeden, infolge von Erdbeben oder anderen unberechenbaren Elementar-Ereignissen direkt oder indirekt entstehenden Schaden in der klaresten und bestimmtesten Form ausschließt und dass ihre Cedenten dahin wirken, dass eine solche Klausel nicht nur in den eigenen Policen, sondern auch in den Policen aller in dem gleichen Gebiet arbeitenden Gesellschaften aufgenommen wird. ${ }^{44}$

Diese etwas umständliche Formulierung zeigt, wie ernst es den Rückversicherern war. Sie wollten unmissverständlich zum Ausdruck bringen, worum es ihnen ging: Um eine Klarstellung des Ausschlusses von Katastrophenschäden in der Feuerversicherung durch Einführung einer weltweit einheitlichen Klausel. Sie hatten eine Standardisierung vor Augen. Nach ihren Vorstellungen musste zunächst ein juristisch einwandfreier Klauseltext entwickelt werden. Diesen Standard sollten dann die Feuerversicherer in exakter Gleichheit in sämtliche Verträge auf aller Welt einfügen. Mit diesem Konzept verbanden die Rückversicherer hohe Erwartungen: Erstens sollte in jedem einzelnen Vertrag klarer als bislang zum Ausdruck kommen, dass Erdbebenschäden vertraglich ausgeschlossen seien. Zweitens suchten sie durch eine massenhafte Anwendung einer einheitlichen, präzisen Ausschlussklausel ihr wirtschaftliches Risiko zu verringern. Drittens zielte ihre Strategie darauf ab, die Rechtslage international zu verändern und mittelfristig den Ausschluss von erdbebenverursachten Schäden als Gewohnheitsrecht zu verankern. Es ging ihnen also um die Verbesserung der Kommunikation, um die Restabilisierung ihrer versicherungstechnischen Grundlagen und um die Verringerung von Rechtsrisiken.

Zahlreiche Feuerversicherer ${ }^{45}$, Wissenschaftler und Journalisten ${ }^{46}$ schlossen sich den Rückversicherern an. Die Gesellschaften wollten und sollten sich durch eine Klau-

42 Zitiert nach: Zur Lage in San Francisco, in: ÖVZ 1906, S. 235.

43 Zur Lage in San Francisco, in: ÖVZ 1906, S. 235; ähnlich L. Fuld, Feuerversicherung und Erdbeben, in: ÖVZ 1906, S. $121 \mathrm{f}$.

44 Zur Katastrophe in San Francisco (Erklärung von 20 europäischen Rückversicherungsgesellschaften), in: Masius‘ Rundschau 1906, S. 227.

45 Z.B. Nachklänge von San Francisco, in: Masius' Rundschau 1906, S. 385; Stronger Earthquake Clauses Advocated, in: The Journal of Commerce vom 12.5.1906, S. 13.

46 L'Argus, zitiert nach: Der Versicherungsfreund vom 1.10.1906, S. 2 f; Noch immer Erdbebengefahr, in: Handel und Industrie, zitiert nach: Der Versicherungsfreund vom 20.2.1907, S. 4; The Earthquake Clause, in: The Adjuster 33, Oktober 1906, S. 189 f.; Earthquake Clause, in: The Journal of Commerce vom 23./24.1.1907, S. 12. 
sel, »an der auch die grösste juristische Spitzfindigkeit nichts zu drehen und zu deuteln finden kann«, von jeder Haftung für indirekte und direkte Feuerschäden freizeichnen. ${ }^{47}$ Die Zeitschrift Handel und Industrie gab sich zuversichtlich:

»Der Wortlaut einer solchen Klausel wird nicht leicht zu finden sein; er muss aber gefunden werden und wird sich schliesslich auch finden lassen, wenn man nur ernstlich danach sucht. $\ll^{48}$

Genau darauf hin wirkten schon 1906 zahlreiche Kräfte. Die Versicherer setzten sich mit dem vielfachen Versagen ihrer Ausschlussklauseln auseinander. Wo lagen die Probleme? Die amerikanischen, deutschen und österreichischen, chilenischen und bald auch jamaikanischen und britischen Gerichte erklärten die Erdbebenklauseln stets für zulässig. Als eine der Schwachstellen erkannten die Versicherer die Verteilung der Beweislast:

»In San Francisco arbeiteten die Versicherten mit der offenen Tatsache, dass es unmöglich war, die Beweise dafür beizubringen, es sei der Brand, der eine bestimmte Proprietät zerstörte, in Wirklichkeit infolge des Erdbebens zum Ausbruch gekommen, und die Gerichtshöfe waren nur zu bereitwillig, sie in dieser Anschauung zu unterstützen. Es lässt sich darum nicht unterschätzen, von welcher grossen Wichtigkeit es ist, dass die Pflicht der Beweisführung den Versicherten auferlegt wird. « ${ }^{49}$

Auch unklare Formulierungen gingen stets zu Lasten der Versicherer. Daher sollte der Ausschluss der Erdbebengefahr eindeutiger als bisher zum Ausdruck kommen. ${ }^{50}$ Diese Erkenntnisse über die prozessuale Tauglichkeit der Erdbebenregelungen waren von ebenso großer Bedeutung wie jene über die materiellrechtlichen Schwachstellen. Sie alle wurden bei der späteren Entwicklung einer neuen Standardklausel aufmerksam berücksichtigt.

Doch noch war die perfekte Erdbebenklausel nicht gefunden. Der Mangel an Informationen führte zu einem Gewirr an Vorschlägen. So empfahl ein deutscher »Fachmann « in der Österreichischen Revue die »Hamburger transatlantischen Polizze ${ }^{51}$, der Brite Mackenzie plädierte für die südamerikanische Klausel der britischen Gesellschaften $^{52}$ und die französische Zeitschrift L'Argus hielt die französische Fassung für die beste ${ }^{53}$.

47 Noch immer Erdbebengefahr, in: Handel und Industrie (wie Fn. 46).

48 Noch immer Erdbebengefahr, in: Handel und Industrie, (wie Fn. 46).

49 R. K. Mackenzie, The San Francisco earthquake \& conflagration (1907).

50 Siehe die Berichte in Wallmann's Versicherungs-Zeitschrift 1907/I, S. 630, Deutsche Versicherungs-Zeitung 1907, S. 4 und Annalen des gesamten Versicherungswesens 1906, S. 32.

51 Der zitierte Versicherer lobte besonders deren Beweislastumkehr. Zur San FranciscoKatastrophe, in: Österreichische Revue 1906, S. 130.

52 R. K. Mackenzie, The San Francisco earthquake \& conflagration (1907). Genauso plädiert eine kalifornische Fachzeitschrift: Couple of Cast Iron Earthquake Clauses, in: The Adjuster 33, Oktober 1906, S. 147.

53 Dabei handelte es sich um eine einfache Ausschlußklausel. Saint Pierre, San Francisco, Valparaiso, in: L'Argus, zitiert nach: Erdbeben und Feuerversicherung, in: Östereichische Revue 1906, S. 231 f.; L'Argus, zitiert nach: Der Versicherungsfreund vom 1.10.1906, S. 2. 


\section{Gefahrausschluss durch Einführung einer einheitlichen Erdbebenklausel}

Wenige Monate nach der San-Francisco-Katastrophe formierten die Generaldirektoren von vier Rückversicherungsgesellschaften eine informelle Arbeitsgruppe, um das Projekt voranzutreiben. ${ }^{54}$ Über ihre Verbindungen zu Feuerversicherungsgesellschaften ließen sie sich Hunderte von Ausschlussklauseln aus aller Welt zusenden. Diese untersuchten sie akribisch und verglichen sie vor dem Hintergrund der Erfahrungen, die sie in San Francisco gemacht hatten. Auf diese Weise gelangten sie zu dem Modell einer Erdbebenklausel, die nach ihrer Einschätzung juristisch unangreifbar war. Bemerkenswert ist dabei, dass sie bei der Entwicklung dieses Standards das staatliche gesetzte Recht in den unterschiedlichen Geschäftsgebieten vollkommen ausblendeten. ${ }^{55}$

Die Implementierung der Klausel überließen die Rückversicherer den Feuerversicherungsgesellschaften in den einzelnen Ländern. Sie verfassten eine umfangreiche Studie mit detaillierten Empfehlungen, die sie allen ihnen bekannten Unternehmen zukommen ließen.$^{56}$ Der hohe Aufwand führte zu einem Erfolg, mit dem sich die Rückversicherer zufrieden zeigten. In zahlreichen europäischen Ländern, von Portugal bis nach ÖsterreichUngarn, übernahmen die Feuerversicherer die Erdbebenklausel modellgetreu in ihre Vertragsbedingungen. Häufig sorgten sie zusätzlich für entsprechende Regelungen in den Gesetzen über den Versicherungsvertrag. Im deutschen Reichstag beispielsweise setzte sich der liberale Abgeordnete Johannes Kaempf mit der Forderung durch, den Ausschluss erdbebenverursachter Schäden gesetzlich festzustellen. ${ }^{57}$ Er überzeugte die Abgeordneten mit der Argumentation, die Regelung in $\S 84$ des künftigen Versicherungsvertragsgesetzes

»[...] würde Deutschland in Übereinstimmung mit den Gesetzentwürfen setzen, die augenblicklich in Frankreich und [...] in Österreich vorliegen und die sogenannte Erdbebenklausel enthalten. Es wäre außerordentlich erwünscht, wenn in dieser Beziehung durch eine gleichartige Gesetzgebung in den verschiedenen Staaten eine internationale Vereinheitlichung der Bestimmung angebahnt würde, und es würde das auch auf die amerikanischen Verhältnisse von günstigem Einfluß sein insofern, als es moralisch dahin drängen würde, dass auch in Amerika Policen und Klauseln, wie ich sie besprochen habe, zur Einführung gelangen. $\ll^{58}$

54 Es handelte sich um die Schweizer Rückversicherungsgesellschaft (Direktor Ch. Simon), die Münchener Rückversicherungsgesellschaft (Dir. C. Thieme), die Kölnische Rückversicherungsgesellschaft (Dir. H. Gruenwald) und die Badische Mit- und Rückversicherungsgesellschaft zu Stuttgart (Dir. B. Lindner).

55 Diese skizzierten Vorgänge konnten aus den Korrespondenzen der Rückversicherer rekonstruiert werden, die im Archiv der Swiss Re (Zürich) sowie vereinzelt bei der Gen Re (Köln) und der Münchener Rück (München) erhalten sind.

56 Die Studie wurde in drei Sprachen veröffentlicht: H. Gruenwald et al., Die Erdbebenklausel in den Versicherungs-Bedingungen der Feuer-Versicherungs-Gesellschaften (Köln 1907); $\mathrm{H}$. Gruenwald et al.: La clause d'exclusion des tremblements de terre dans les conditions de polices des Compagnies d'assurances contre l'Incendie (Zürich 1907); H. Gruenwald et al.: The Earthquake Clause in the Policy-Conditions of the Fire Insurance Companies (Köln 1907). Exemplare aller drei Versionen sind im Archiv der Swiss Re (Zürich) erhalten.

57 Kaempf handelte insgeheim als Mitglied des Aufsichtsrates der Münchener Rück, die in San Francisco die immense Summe von 11 Millionen Mark verloren hatte.

58 Stenographische Berichte über die Verhandlungen des Reichstags, XII. Legislaturperiode, I. Session 1907/1908, S. 1824 f. 
Die Harmonisierung des Rechts in Europa erfolgte also nicht nur um ihrer selbst willen, sondern auch mit Blick auf die USA, wo europäische Versicherungsgesellschaften hoch aktiv waren ${ }^{59}$ und weitere Katastrophen befürchteten. Doch ganz so weit reichte die Fernwirkung der deutschen Gesetzgebung nicht.

\section{Risikoübernahme durch Prämienerhöhung}

Seine Grenzen fand der Standardisierungsprozess bereits in Großbritannien und Holland. Drei Faktoren trugen in diesen Ländern und in den USA zur Ablehnung der Klausel bei: Erstens die Auffassung, man könne erdbebenverursachte Feuerschäden durchaus gegen Zusatzprämien versichern; zweitens der Druck der Öffentlichkeit, die sich insbesondere in den USA gegen die Freizeichnung von Katastrophenschäden verwehrte; und drittens der Druck des Marktes, der sich aufbaute, nachdem einzelne Versicherer die Übernahme des Erdbeben-Feuer-Risikos als Werbefaktor entdeckt hatten. ${ }^{60}$

\section{Globales Risikomanagement avant la lettre}

Als wichtigstes Ergebnis dieser Betrachtungen ist festzuhalten, dass globales Risikomanagement seitens der Privatwirtschaft zu erheblichen Veränderungen des Rechts führen kann.

Neu ist im Jahr 1906 die Erkenntnis, dass globale Risiken nur global zu bewältigen sind. Die Versicherungswirtschaft, allen voran die großen Rückversicherungsgesellschaften in Deutschland und der Schweiz, übten sich erstmals in »globalem Risikomanagement«. Möglich wurde dies vor allem durch das Erstarken der Rückversicherungsbranche ab den 1880er Jahren. Die beteiligten Unternehmen verfügten über Verbindungen zu Direktversicherern auf sämtlichen Kontinenten. Vergleichbare, operativ nutzbare Netzwerke hatten bis dahin nicht existiert. Die herausragende Bedeutung der Rückversicherungsgesellschaften als Motoren der Veränderung des Vertragsrechts nach der San-Francisco-Katastrophe hat ihre Fortsetzung bei dem Management anderer globaler Risiken gefunden. ${ }^{61}$

Zugleich tritt mit der - zumindest teilweise erfolgreichen - Einführung einer einheitlichen Erdbebenklausel eine neuartige Form der Rechtsbildung zutage. Die Wirtschaft vertraut nicht auf das staatliche Recht, sondern agiert und reagiert zunehmend autonom. In vielen Wirtschaftszweigen kommt es zur Bildung von transnationalen Strukturen aus Standardverträgen und -klauseln. Zu nennen sind in erster Linie Transportwirtschaft und Großhandel, Versicherungs- und Finanzwirtschaft. Zwar entsteht noch keine neue lex mercatoria, aber der Prozess weist in diese Richtung. ${ }^{62}$

59 P. Borscheid: Internationalisierung der deutschen Versicherungswirtschaft 1870-1945, in: Vierteljahresschrift für Sozial- und Wirtschaftsgeschichte 88 (2001), S. $311 \mathrm{ff}$.

60 Die Dissertation des Verfassers umfasst eine Analyse der Entwicklungen in den einzelnen Ländern (siehe Fn. 3).

61 Kontinuität zeigt sich sogar auf der Ebene der Unternehmen. Schweizerische, Münchener und Kölnische Rück waren Anfang des 20. Jahrhunderts Marktführer im Rückversicherungswesen und sind es bis heute als Swiss Re, Munich Re und Gen Re.

62 Eine vertiefte Auseinandersetzung mit der Rechtsbildung durch Standardisierung von Vertragsinhalten erscheint in der Dissertation des Verfassers (siehe Fn. 3). 
Die Untersuchung der Reaktionen der Versicherungswirtschaft auf die ErdbebenFeuer-Katastrophe von San Francisco zeigt darüber hinaus, wie unterschiedlich bestimmte Schadensereignisse und die mit ihnen verbundenen Risiken bzw. Gefahren wahrgenommen werden. Im Beispielsfall beruhten diese Unterschiede vor allem auf einem transatlantischen Gefälle des gesicherten Wissens über die Ursachen von Erdbeben. Doch auch andere Faktoren wie der kulturelle Hintergrund oder religiöse Vorstellungen können zu unterschiedlichen Interpretationen von Katastrophenereignissen führen. In der internationalen Versicherungswirtschaft sind allerdings erhebliche Differenzen bei der Einschätzung bestimmter Wahrscheinlichkeiten durch eine Zunahme des gegenseitigen Informationsaustausches inzwischen selten geworden.

Bemerkenswert ist, wie stark die Wahrnehmung bestimmter Ereignisse und Entwicklungen die Einschätzung der Risiken oder Gefahren und die Reaktionen der Versicherungswirtschaft prägte und prägt. Im hiesigen Beispielsfall ist zu beobachten, dass die Versicherer bei der Formulierung der Erdbebenklausel sämtliche gerichtlichen Erfahrungen mit den bis dahin verwendeten Freizeichungsklauseln akribisch berücksichtigten. So achteten sie besonders darauf, dass die Beweislast für das Vorliegen einer Ausnahme von der Haftung von ihren Schultern auf die Versicherungsnehmer übertragen wurde. ${ }^{63}$ Dementsprechend lautete beispielsweise der neue $\S 1$ Abs. 4 der ab dem 1. Januar 1910 für alle deutschen Feuerversicherungsgesellschaften verbindlichen Vertragsbedingungen:

»Im Falle von inneren Unruhen oder Kriegsereignissen jeder Art sowie im Falle von Erdbeben haftet der Versicherer nur, wenn der Versicherungsnehmer nachweist, daß der Schaden mit diesen Ereignissen weder unmittelbar noch mittelbar in Zusammenhang steht. ${ }^{64}$

63 H. Gruenwald et al., Die Erdbebenklausel in den Versicherungs-Bedingungen der FeuerVersicherungs-Gesellschaften (Köln 1907), S. 9.

64 Die gesamte Ausschlussregelung in den AVB der Vereinigung der in Deutschland arbeitenden Privat-Feuerversicherungsgesellschaften und des Verbandes Deutscher Privat-Feuerversicherungsgesellschaften von 1909, nach Genehmigung des Reichsaufsichtsamtes [Senatsentscheidung vom 26.8.1909, VA 1909, 266, vgl. 155] in Kraft getreten am 1.1.1910, lautet nach dem Kommentar zu den Allgemeinen Feuerversicherungsbedingungen von R. Raiser, S. 1 f., 18, 42: »§ 1 (1) Der Versicherer haftet nach Maßgabe der nachfolgenden Bestimmungen für den Schaden, der an den versicherten Sachen durch Brand, Blitzschlag oder durch Explosion von Leuchtgas aller Art, auch wenn es zu anderen als Beleuchtungszwecken dient, oder durch Explosion von Haushaltungs-Heizungseinrichtungen und von Beleuchtungskörpern entsteht. Die Haftung des Versicherers für den durch Explosionen anderer Art entstehenden Schaden bedarf der besonderen Vereinbarung.

(2) Im Falle eines Brandes hat der Versicherer den durch die Zerstörung oder Beschädigung entstehenden Schaden zu ersetzen, soweit der Schaden die versicherten Sachen betrifft, welche zerstört oder beschädigt werden, und soweit die Zerstörung oder die Beschädigung auf der Einwirkung des Feuers beruht oder bei dem Brande durch Löschen, Niederreißen oder Ausräumen verursacht wird, oder die unvermeidliche Folge eines Brandes ist, der auf dem Grundstücke, auf welchem sich die versicherten Sachen befinden, oder einem angrenzenden Nachbargrundstücke stattgefunden hat. Der Versicherer hat auch den Wert der versicherten Sachen zu ersetzen, welche bei dem Brande abhanden kommen. Einen weiteren Schaden, insbesondere einen weiteren mittelbaren Schaden, sowie den durch Eintritt des Versicherungsfalls entgehenden Gewinn umfaßt die Versicherung nur, soweit dies besonders vereinbart ist. (3) [Haftungsumfang bei Blitzschlag und Explosion]

(4) Im Falle von inneren Unruhen oder Kriegsereignissen jeder Art sowie im Falle von Erdbeben haftet der Versicherer nur, wenn der Versicherungsnehmer nachweist, daß der Schaden mit diesen Ereignissen weder unmittelbar noch mittelbar in Zusammenhang steht.

(5) [Haftung nur bei Brandereignissen]«. 
Die Beschreibungen und Begrenzungen von Risiken fanden aber schon damals ihre Grenzen in den sprachlichen Möglichkeiten, die den Versicherern und Juristen zur Verfügung standen. Dies galt und gilt in erhöhtem Maße, wenn einheitliche Regelungen über Länder- und Sprachgrenzen hinweg eingeführt werden sollen. Nach der SanFrancisco-Katastrophe verzichteten die beteiligten Rückversicherungsdirektoren darauf, die von ihnen gesuchte »perfekte« Erdbebenklausel auszuformulieren. Statt dessen begnügten sie sich mit der Nennung einzelner Elemente der künftigen Klausel und überließen die Umsetzung den Direktversicherern. ${ }^{65}$ Diese fanden - sofern sie neue Erdbebenklauseln einzuführen bereit waren - so ähnliche Lösungen, dass bei einer Gesamtbetrachtung von einer internationalen Standardisierung gesprochen werden kann.

65 Dies zeigt sich an der Diskussion, die der Versendung ihrer Empfehlungen unmittlbar vorausging: Schreiben von Simon (Schweizerische Rück) an Herlitz (Skandia Rückv.-G., Stockholm) vom 3.5.1907, in: SR/A SR/A 9.0-32/ gelber Ordner (Korresp., v.a. Kölnische Rück 1907), Bl. 33 f.; Schreiben von Lindner (Badische Mit- und Rückv.-G.) an Grünwald (Kölnische Rückv.-G.) vom 7.5.1907, in: SR/A a.a.O., Bl. 52 ff.; Schreiben von Grünwald an Thieme (Münchener Rückv.-G.), Lindner und Simon vom 27.4.1907, in: SR/A a.a.O., B1. $8 \mathrm{f}$. 